Hendrik C. Kuhlmann

\title{
Thermocapillary \\ Convection \\ in Models \\ of Crystal Growth
}

With 101 Figures 


\section{Contents}

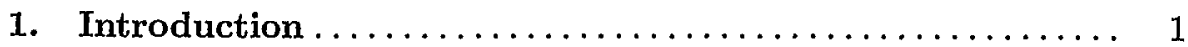

1.1 Marangoni Effect ........................... 1

1.2 Thermocapillary Convection in Crystal Growth .......... 3

1.3 Previous Reviews .......................... 5

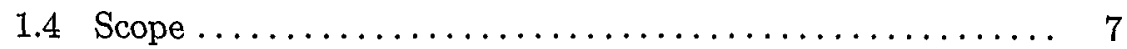

2. Basic Equations $\ldots \ldots \ldots \ldots \ldots \ldots \ldots \ldots \ldots \ldots \ldots \ldots \ldots \ldots \ldots \ldots$

2.1 Volume Equations ........................... 11

2.2 Boundary Conditions $\ldots \ldots \ldots \ldots \ldots \ldots \ldots \ldots \ldots \ldots, 11$

2.2.1 Velocity Field .......................... 11

2.2 .2 Temperature Field $\ldots \ldots \ldots \ldots \ldots \ldots \ldots \ldots \ldots, 12$

2.3 Dimensionless Equations . . . . . . . . . . . . . . . . 13

3. Asymptotic Behavior ......................... 19

3.1 Flow Near the Contact Line $\ldots \ldots \ldots \ldots \ldots \ldots \ldots \ldots \ldots . \ldots \ldots$

3.2 Boundary-Layer Scalings ..................... 25

3.2.1 Low-Prandtl-Number Inertial Flow ............ 26

3.2.2 Boundary Layers Near the Cold Corner .......... 27

3.2.3 High-Prandtl-Number Thermocapillary Flow in a Hot Corner (Viscous-Convective Limit) ....... 30

4. Creeping Flow in Thermocapillary Liquid Bridges ....... 35

4.1 Method of Biorthogonal Functions ................. 36

4.2 Thermocapillary Flow and Small Surface Deformations $\ldots \ldots \ldots \ldots \ldots \ldots \ldots \ldots, 40$

4.2 .1 Formulation $\ldots \ldots \ldots \ldots \ldots \ldots \ldots \ldots \ldots \ldots, 40$

4.2 .2 Flow Field $\ldots \ldots \ldots \ldots \ldots \ldots \ldots \ldots \ldots \ldots \ldots, 41$

4.2 .3 Temperature Field...................... 42

4.2.4 Dynamic Surface Deformations............... 43

4.2.5 Flow Patterns............................ 44

4.2 .6 Aspect Ratio Limits ..................... 49

4.3 Axial Through Flow ....................... 51 
5. Concepts of Stability Analysis $\ldots \ldots \ldots \ldots \ldots \ldots \ldots \ldots \ldots, 59$

5.1 Linear Stability ............................ 59

5.1.1 Temporal and Spatial Stability ............... 61

5.1 .2 Convective and Absolute Stability ............ 62

5.2 Energy Analysis . ........................... 64

5.2.1 Reynolds-Orr Equation .................. 64

5.2.2 Energy Equations for Infinitesimal Perturbations ..... 66

5.3 Energy Stability ........................... 67

5.4 A Posteriori Energy Balances ................... 69

6. Plane Thermocapillary Layers .................. 71

6.1 Convective Stability of the Return Flow ............ 71

6.2 Asymptotics of the Critical Values for $\operatorname{Pr} \rightarrow 0 \ldots \ldots \ldots \ldots .80$

7. Convection in Cylindrical Geometry ............. 83

7.1 Axisymmetric Convection in Liquid Bridges............ 83

7.2 Linear Stability Analysis $\ldots \ldots \ldots \ldots \ldots \ldots \ldots \ldots \ldots . \ldots 9$

7.3 Buoyant Instability . . . . . . . . . . . . . . . . . . 91

7.3.1 Small Prandtl Numbers: $\operatorname{Pr}=0.02 \ldots \ldots \ldots \ldots \ldots . . . \ldots 5$

7.3.2 Large Prandtl Numbers: $\operatorname{Pr}=1 \ldots \ldots \ldots \ldots \ldots \ldots . \ldots 9$

7.4 Thermocapillary Instability ................... 104

7.4.1 Small Prandtl Numbers: $\operatorname{Pr}=0.02 \ldots \ldots \ldots \ldots \ldots . \ldots 107$

7.4.2 Large Prandtl Numbers: $\operatorname{Pr}=4 \ldots \ldots \ldots \ldots \ldots \ldots 111$

7.4.3 Remarks on the Stability

of Pure Thermocapillary Flows ............... 117

7.5 Instability of Mixed Convection .................. 119

7.5.1 Thermocapillarity Dominating ............... 119

7.5.2 Buoyancy Dominating ................... 123

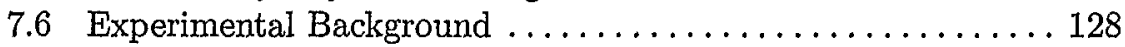

7.7 Three-Dimensional Simulations . . . . . . . . . . . . . . . 132

7.7.1 Low Prandtl Numbers ..................... 133

7.7.2 High Prandtl Numbers .................... 135

7.8 Energy Stability Analyses $\ldots \ldots \ldots \ldots \ldots \ldots \ldots \ldots \ldots . \ldots \ldots$

8. Convection in Rectangular Geometry ................ 143

8.1 Two-Dimensional Flows ........................ 145

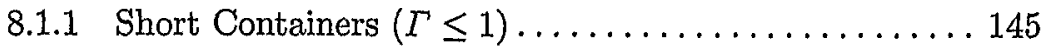

8.1.2 Stationary Flow in Long Containers $(\Gamma \gg 1) \ldots \ldots \ldots 147$

8.1.3 Two-Dimensional Oscillatory Flows ............. 151

8.2 Three-Dimensional Flows . . . . . . . . . . . . . . . . . . . 154

8.2.1 Large Aspect Ratios ....................... 154

8.2 .2 Side-wall Effects......................... 154

8.2.3 Steady and Spatially Periodic

Three-Dimensional Flows ................... 158 
9. Extensions to Simple Models . . . . . . . . . . . . . . 163

9.1 Intrinsic Properties of Thermocapillary Systems ......... 163

9.1.1 Static Free-Surface Shape .................. 163

9.1.2 Dynamic Surface Deformations................ 168

9.1.3 Non-Monotonic Surface Temperature Profiles ....... 169

9.1.4 Moving Liquid-Solid Interfaces................ 171

9.1.5 Temperature-Dependent Material Parameters ....... 173

9.1.6 Binary Mixtures and Adsorption .............. 174

9.1.7 External Convection: Double Layers . . . . . . . . . . 178

9.2 External Body Forces ........................ 180

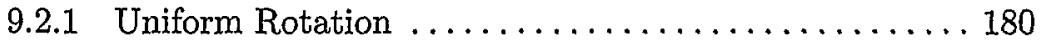

9.2 .2 Vibrations............................ 182

9.2.3 Magnetic Fields ........................ 185

A. Appendix .............................. 191

A.1 Some Material Parameters ...................... 191

A.2 Stokes Flow Near the Contact Line ................ 192

A.3 Biorthogonal Functions for Oseen-Type Flows ............ 194

A.4 Chebyshev-Galerkin Method for Plane Layers............ 201

References................................. 203

Index $\ldots \ldots \ldots \ldots \ldots \ldots \ldots \ldots \ldots \ldots \ldots \ldots \ldots \ldots \ldots \ldots \ldots \ldots \ldots \ldots \ldots, 221$ 